\title{
DNA methylation profiling analysis identifies a DNA methylation signature for predicting prognosis and recurrence of lung adenocarcinoma
}

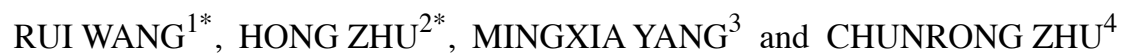 \\ ${ }^{1}$ Department of Oncology, Anhui Chest Hospital, Hefei, Anhui 230022; ${ }^{2}$ Department of Internal Medicine Oncology, \\ The First Affiliated Hospital of Soochow University, Suzhou, Jiangsu 215006; ${ }^{3}$ Department of Pulmonary and \\ Critical Care Medicine, The Affiliated Changzhou No. 2 People's Hospital, Changzhou, Jiangsu 213002; \\ ${ }^{4}$ Department of Oncology, The First Affiliated Hospital of Soochow University, Suzhou, Jiangsu 215006, P.R. China
}

Received December 12, 2018; Accepted August 16, 2019

DOI: $10.3892 / \mathrm{ol} .2019 .10931$

\begin{abstract}
The aim of the present study was to identify candidate prognostic DNA methylation biomarkers for lung adenocarcinoma (LUAD), since the modern precise medicine for the treatment of LUAD requires more biomarkers and novel therapeutic targets of interest. DNA methylation profiling data of LUAD were downloaded from The Cancer Gene Atlas portal. Differentially methylated genes (DMGs) were screened to differentiate between samples designated as good and bad prognosis. LUAD-associated methylation modules were obtained with the weighted correlation network analysis (WGCNA) package, followed by function enrichment analysis. Optimal prognostic DMGs were selected using the LASSO estimation-based Cox-PH approach and were used to construct a prognostic risk scoring system. The training set was dichotomized by risk score, into high- and low-risk groups. The
\end{abstract}

Correspondence to: Dr Mingxia Yang, Department of Pulmonary and Critical Care Medicine, The Affiliated Changzhou No. 2 People's Hospital, 29 Xinglong Lane, Changzhou, Jiangsu 213002, P.R. China

E-mail: cougermx@126.com

Dr Chunrong Zhu, Department of Oncology, The First Affiliated Hospital of Soochow University, 188 Shizi Street, Suzhou, Jiangsu 215006, P.R. China

E-mail: medjssz@163.com

*Contributed equally

Abbreviations: LUAD, lung adenocarcinoma; DMGs, differentially methylated genes; WGCNA, weighted correlation network analysis; OS, overall survival; RFS, recurrence-free survival; NSCLC, non-small-cell lung cancer; TCGA, The Cancer Genome Atlas; $\mathrm{PH}$, proportional hazards; TOM, topological overlap matrix; PCC, Pearson correlation coefficient

Key words: DNA methylation, prognosis, recurrence, WGCNA, risk score differences in overall survival (OS) time or recurrence-free survival (RFS) time between the two groups were evaluated using a Kaplan-Meier curve. A total of 742 DMG samples were screened for good and bad prognosis. WGCNA identified three LUAD-associated modules, which were primarily associated with cytoskeleton organization, transcription and apoptosis. A nine-gene prognostic methylation signature was determined, which included C20orf56, BTG2, C13orf16, DNASE1L1, ZDHHC3, FHDC1, ARF6, ITGB3 and ICAM4. A risk score-based methylation signature classified the patients in the training set into high- and low-risk groups with significantly different OS or RFS times. The prognostic value of the methylation signature was successfully verified in a validation set. In conclusion, the present study identified a nine-gene methylation signature for the prediction of survival and recurrence in patients with LUAD and improved the understanding of the alterations in DNA methylation in LUAD.

\section{Introduction}

Lung adenocarcinoma (LUAD) is the most predominant subtype of non-small cell lung cancer (NSCLC), with increased incidence over the past decades worldwide $(1,2)$. LUAD is usually observed in the peripheral region of the lungs, with a poor overall five-year survival rate of $15 \%$ worldwide in 2008 (3). Due to resistance to radiation therapy, LUAD is often treated surgically (4). Nonetheless, approximately a third of patients relapse within five years of surgical removal (5).

DNA methylation, a primary epigenetic modification in the mammalian genome, often occurs at $\mathrm{CpG}$ islands, leading to altered tumor suppressor gene transcription (6). Aberrant DNA methylation plays a key role in the progression and metastasis of LUAD, reflecting important biological features in the etiology (7). Zhu et al (8) identified a group of genes with differentially methylated loci in LUAD. Han et al (9) found that methylated PTPRF (protein tyrosine phosphatase, receptor type F), HOXD3 (homeobox D 3), HOXD13 and CACNA1A (calcium voltage-gated channel subunit alpha1 A) may be potential markers of LUAD, based on DNA methylation profiling analysis. Furthermore, Sandoval et al (10) suggested 
a prognostic signature based on five hypermethylated genes for early stage NSCLC. Additionally, Kuo et al (11) established a proof-of-concept prognostic signature of eight methylated probes for survival prediction in Asian and Caucasian populations with early-stage LUAD. Despite these remarkable findings, there is a lack of a prognostic DNA methylation signature for LUAD.

In the present study, genome-wide methylation analysis was carried out on the methylation data of 425 patients with LUAD, with corresponding clinicopathological features from The Cancer Genome Atlas (TCGA). LUAD-associated co-methylation modules were mined with the weighted correlation network analysis (WGCNA) package. Furthermore, a group of differentially methylated genes (DMGs) predictive of survival were identified for LUAD by performing differential DNA methylation, correlation, univariate Cox regression and L1 penalized (LASSO) Cox proportional hazards $(\mathrm{PH})$ regression analyses. These findings may potentially contribute to a deeper insight into the epigenetic landscape of LUAD and improve the prognostic evaluation of patients.

\section{Materials and methods}

Data resources. DNA methylation data and the corresponding survival information of 425 LUAD tissue samples were downloaded from TCGA portal (https://gdc-portal.nci.nih. gov/) on May 26th, 2018, based on the Illumina Infinium Human Methylation 450 BeadChip platform, and were used as the training set in the present study. The GSE39279 dataset was downloaded from the National Center for Biotechnology Information Gene Expression Omnibus (NCBI GEO) database (http://www.ncbi.nlm.nih.gov/geo/), based on the Illumina Infinium Human Methylation 450 BeadChip platform, consisting of the gene methylation data of 443 NSCLC samples. Among these samples, 155 LUAD samples with available survival information were selected as the validation set. The clinicopathological features of the training set and the validation set are shown in Table I.

Differential DNA methylation analysis. A bad prognosis was defined as patients who died or who had a survival time $<12$ months, whereas a good prognosis indicated living patients who survived $>24$ months. According to the annotation profiles provided by the platform, only the DNA methylation loci in CpGs were reserved. The comparison of samples associated with good and bad prognosis from the TCGA set, identified differentially methylated CpGs (DM CpGs), using the limma package (12) of R language (version 3.34.7; https://bioconductor.org/packages/release/bioc/html/limma.html). A strict cut-off value was set at false discovery rate (FDR) $<0.05$ and $\mid \log _{2}$ fold change (FC) $\mid>0.1$. The genes mapped by the identified DM CpGs were defined as the DMGs.

Co-methylation analysis. In order to analyze the inter-correlation among the identified DM CpGs, co-methylation network analysis was carried out using the WGCNA R package (version 1.63; https://cran.r-project. org/web/packages/WGCNA/index.html), as previously described (13). Briefly, a thresholding power function $(\beta)$ of
Table I. Clinicopathological characteristics of patients in the training set and the validation set.

\begin{tabular}{lcc}
\hline $\begin{array}{l}\text { Clinicopathological } \\
\text { characteristics }\end{array}$ & $\begin{array}{c}\text { Training set } \\
(\mathrm{n}=425)\end{array}$ & $\begin{array}{c}\text { Validation set } \\
(\mathrm{n}=155)\end{array}$ \\
\hline $\begin{array}{l}\text { Age, years (mean } \pm \mathrm{SD}) \\
\text { Sex, male/female }\end{array}$ & $65.12 \pm 10.04$ & $65.11 \pm 10.85$ \\
$\begin{array}{l}\text { Death, dead/alive/- } \\
\text { OS time, months } \\
\text { (mean } \pm \text { SD) }\end{array}$ & $198 / 227$ & $76 / 79$ \\
$\begin{array}{l}\text { RFS time, months } \\
\text { (mean } \pm \text { SD) }\end{array}$ & $18.802 \pm 26.34$ & - \\
Recurrence, yes/no & $87 / 228$ & - \\
\hline
\end{tabular}

SD, standard deviation; -, information unavailable; OS, overall survival; RFS, recurrence-free survival.

5 was chosen to fit a scale-free network. Topological overlap matrix (TOM) was then calculated to measure the correlations between the methylation levels of two genes. The resulting hierarchical clustering dendrogram was obtained, followed by selection of the modules with a minimum module size of 100 and a minimum cut height of 0.95 , using the Dynamic Tree Cut algorithm. These identified DM CpGs were then mapped to the modules obtained by WGCNA analysis. The enrichment of target DM CpGs in each module was assessed by hypergeometric-based test (14), using the following formula: $\mathrm{f}(\mathrm{k}, \mathrm{N}, \mathrm{M}, \mathrm{n})=\mathrm{C}(\mathrm{k}, \mathrm{M}) \times \mathrm{C}(\mathrm{n}-\mathrm{k}, \mathrm{N}-\mathrm{M}) / \mathrm{C}(\mathrm{n}, \mathrm{N})$.

The modules with $\mathrm{P}<0.05$ and fold enrichment $>1$ were further selected as LUAD-associated modules and subjected to Gene ontology (GO) enrichment analysis using the DAVID 6.8 software $(15,16)$. This revealed the biological functions of the DMGs clustered in these modules.

Correlation of DNA methylation level with gene expression level. The genome-wide expression data of the LUAD samples in the TCGA set was obtained. The correlation between the overall methylation level and the overall gene expression level of the DMGs, included in the LUAD-associated modules, was analyzed with the cor.test function of R language by calculating the Pearson's correlation coefficient (PCC) (17). Subsequently, the PCC of the overall methylation level of each individual gene, with its overall gene expression level was also computed. The genes with negative $\mathrm{PCC}$ and $\mathrm{P}<0.05$ were used for further analysis.

Construction of a prognostic risk scoring model based on the training set. Univariate Cox regression analysis was performed to identify the prognosis-associated DMGs from the aforementioned genes with negative PCC, using the survival package of R language (18) (http://bioconductor.org/packages/survivalr/), with $\log$-rank $\mathrm{P}<0.05$ as the cutoff.

A LASSO estimation-based Cox-PH model (19) was used to select the optimal panel of genes predictive of prognosis from these prognosis-related DMGs by the penalized package (version 0.9-50) (20) of R language. Combining the Cox-PH coefficients of the optimal genes with their methylation levels, 
A
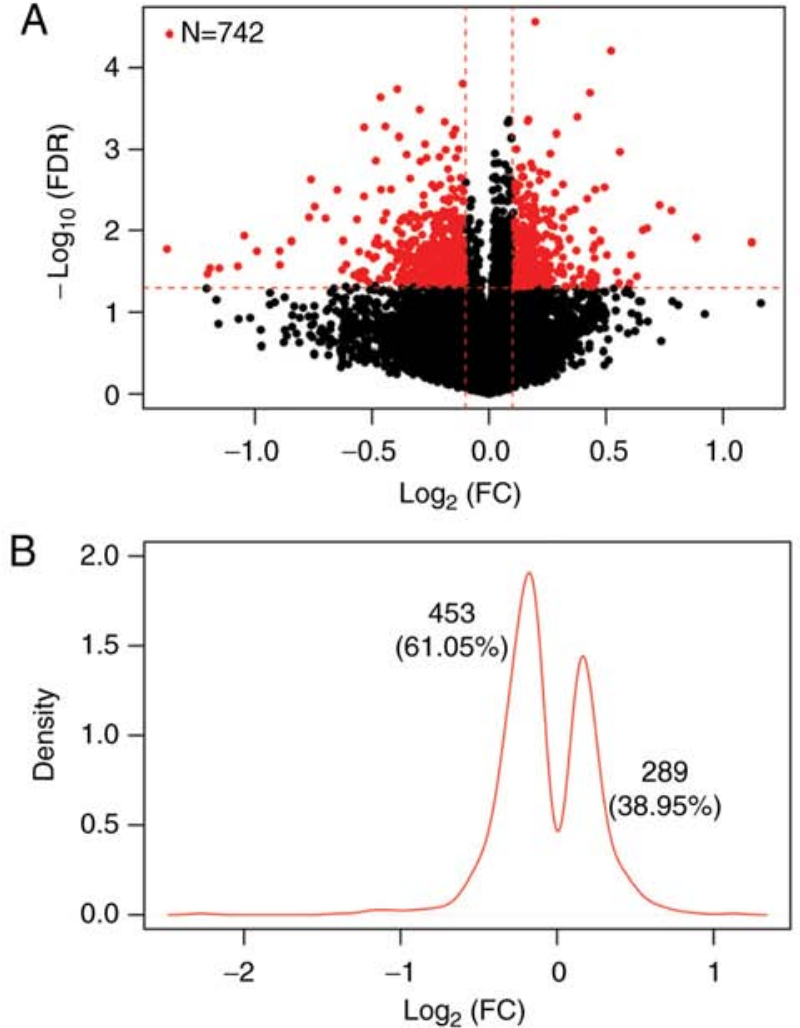

C Bad prognostic

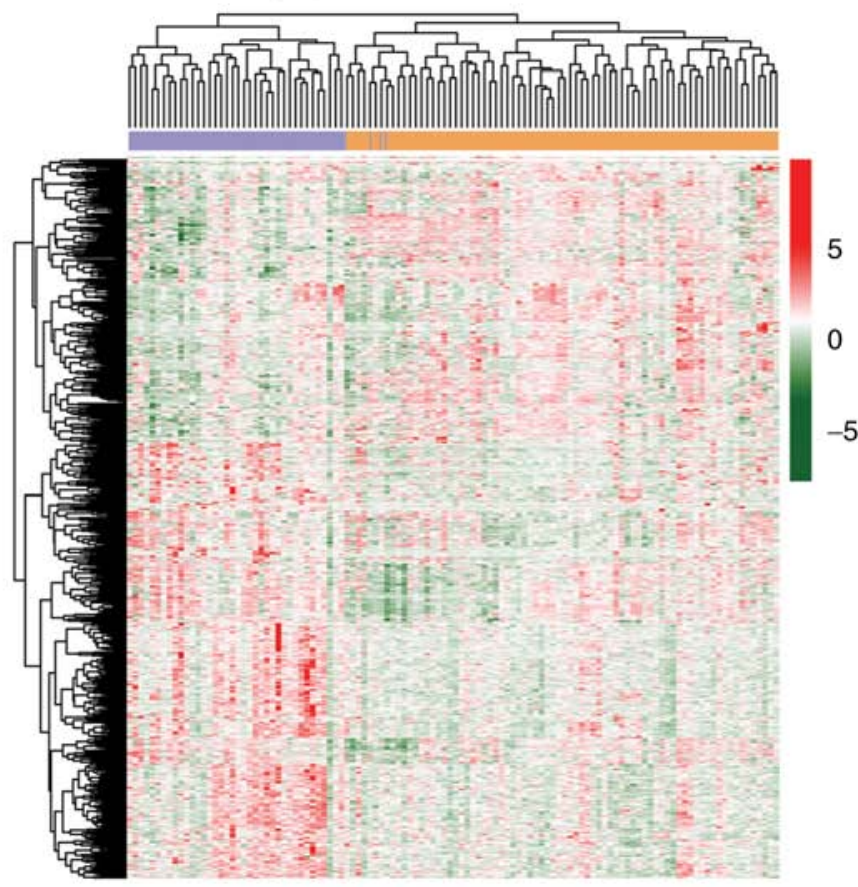

Figure 1. Analysis of DMGs between samples with good and bad prognosis. (A) Volcano plot of effect size $\log _{2}(\mathrm{FC})$ against - $\log _{10}$ (FDR) of DMGs. Red spots represent DMGs with FDR $<0.05$ (horizontal red dashed line) and $\log _{2} \mathrm{FCl}>0.1$ (two vertical red dashed lines). (B) Kernel density plotting of $\log _{2}(\mathrm{FC}) \mathrm{displays}$ 289 DMGs of hypermethylation (61.05\%) and 453 DMGs of hypomethylation (38.95\%). (C) Two-way hierarchical clustering analysis of the samples in the The Cancer Genome Atlas set, using a heatmap based on the methylation level of the DM CpGs. The color bar represents CpG methylation level (green to red, low to high level). FC, fold changes; FDR, false discovery rate; DMGs, differentially methylated genes.

a risk scoring model was constructed for predicting survival as follows: Risk score $=\sum$ coef $_{\text {gene }} \mathrm{x}$ methylation ${ }_{\text {gene }}$, where coef $_{\text {gene }}$ represents the Cox-PH coefficient of a gene and methylation ${ }_{\text {gene }}$ represents the methylation level of a gene.

A risk score was assigned to each patient in TCGA dataset and the median risk score for all patients was calculated to divide patients into a low-risk group (risk score below the median value) and a high-risk group (risk score above the median value). To estimate the overall survival (OS) time and recurrence-free survival (RFS) time of the patients in the two risk groups, the Kaplan-Meier estimate (21) was used together with the Wilcoxon log rank test. The areas under the receiver operating characteristic (AUROC) curves were used to evaluate the prognostic ability of the risk scoring model and tested in the validation set.

\section{Results}

$D M C p G s$ between samples with good and bad prognosis. With regard to the DNA methylation data of the TCGA set, 15654 methylated loci in CpGs were retained, according to the Illumina $450 \mathrm{~K}$ methylation platform. This set was comprised of 41 samples with bad prognosis and 72 samples with good prognosis. As shown in Fig. 1A, a total of 742 DMGs $\left(3.79 \times 10^{-7}\right.$ $<$ P-nominal $<6.84 \times 10^{-4}, 2.77 \times 10^{-5}<$ FDR $\left.<0.05\right)$ were identified between the samples with good and bad prognosis. Kernel density plotting of $\log _{2} \mathrm{FC}$ showed that of the 742 DMGs, 289 were hypermethylated, whereas 453 were hypomethylated in the samples with good prognosis (Fig. 1B). Two-way hierarchical clustering analysis based on the methylation levels of these DMGs revealed that the subjects in the TCGA set were clustered into two different groups (Fig. 1C). Furthermore, out of the CpGs of the 742 identified DMGs, 447 were located in gene bodies, 155 in 5' untranslated regions (UTRs), 98 in 3'UTRs and 42 in promoters. The top 20 DMGs were chosen according to their FDR value and shown in ascending order in Table II.

LUAD-associated co-methylation modules. For the purpose of detecting LUAD-associated co-methylation modules, a weighted gene co-methylation network was constructed for the identified DMGs. As depicted in Fig. 2A and B, the DMGs with significantly correlated methylation level $(\mathrm{P}<0.05)$ were assigned into 13 methylation modules (Table III). The DMGs without significant correlation in their methylation level were grouped into the grey module.

The enrichment of the DMGs in each module was analyzed using a hypergeometric-based test. Three modules with fold enrichment $>1$ and $\mathrm{P}<0.05$ were identified as LUAD-associated methylation modules (black module, size $=68$; pink module, size $=27$; salmon module, size=26; Fig. 2C). Moreover, GO enrichment analysis found that the total of 121 DMGs in the three modules were highly enriched in 16 biological processes, primarily associated with cell death, cytoskeleton organization and cell junction organization (Table IV). 


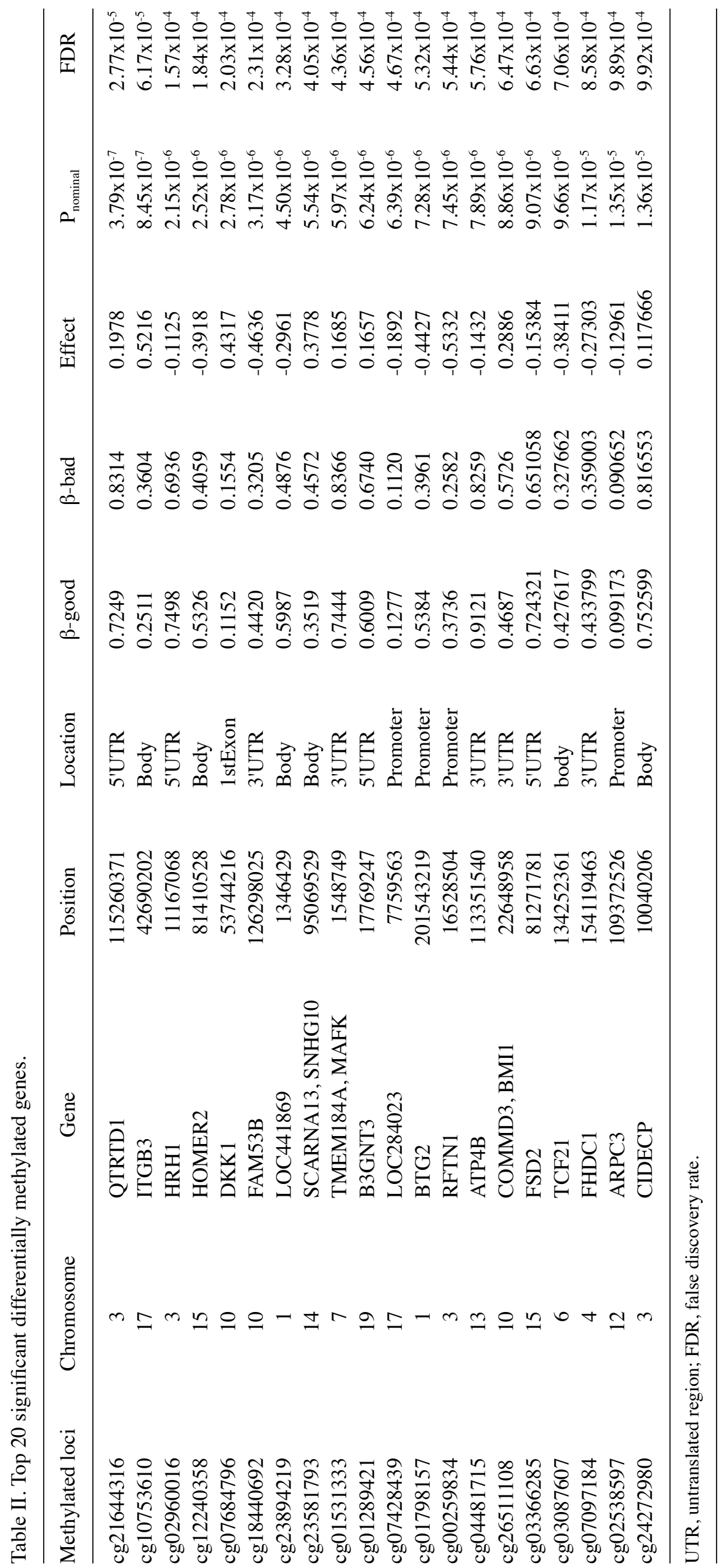



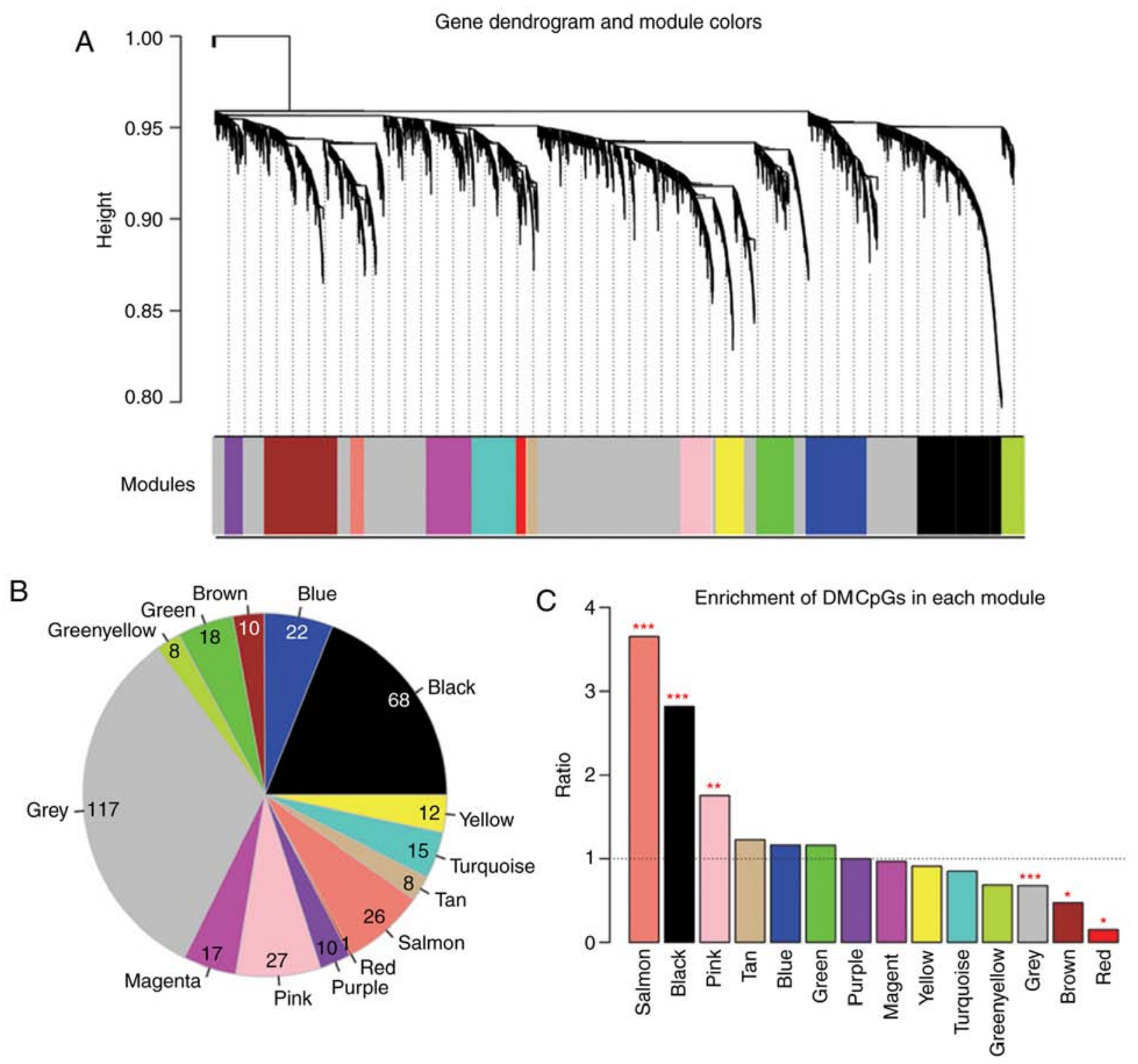

Figure 2. WGCNA methylation modules. (A) A hierarchical clustering dendrogram generated by WGCNA analysis. A total of 14 modules were identified and depicted in different colors. The colored row underneath shows the module assignment determined by the Dynamic Tree Cut. (B) The number of differentially methylated genes mapped to different modules. (C) Fold enrichment of each module. Fold enrichment represents enrichment of differentially methylated CpGs in each module. ${ }^{*} \mathrm{P}<0.05,{ }^{* *} \mathrm{P}<0.01$ and ${ }^{* * * *} \mathrm{P}<0.001$. WGCNA, weighted correlation network analysis.

Development and validation of a prognostic scoring model based on DMGs. The methylation levels of the 121 DMGs clustered in the three LUAD-associated modules were negatively correlated with their overall gene expression levels ( $\mathrm{PCC}=-0.5008, \mathrm{P}=1.618 \times 10^{-8}$; Fig. 3). Furthermore, the negative correlation between the methylation level of the individual gene and its gene expression level was observed in 94 genes, of which 58 DMGs were found to be significantly associated with survival via univariate Cox regression analysis $(\mathrm{P}<0.05$, Table V). Consequently, a LASSO Cox PH model was fitted with the methylation level of the 58 prognosis-associated DMGs. When the cross-validated likelihood (CVL) reached the maximal value of 1.537, the optimal $\lambda$ value was -691.265 . As a result, the most powerful prognostic panel of genes was selected, including $C 20$ orf56, BTG anti-proliferation factor 2 (BTG2), C13orf16, deoxyribonuclease 1 Like 1 (DNASE1L1), zinc finger DHHC-type containing 3 (ZDHHC3), FH2 domain containing 1 (FHDC1), ADP ribosylation factor 6 (ARF6), integrin subunit beta 3 (ITGB3) and intercellular adhesion molecule 4 (ICAM4) (Table VI).

The association of the methylation level of the nine prognostic genes and survival was investigated. All samples in the TCGA set were dichotomized into hypermethylated group and hypomethylated group, based on the median methylation level of each prognostic gene, separately (Fig. 4). For C20orf56, BTG2, ZDHHC3, FHDC1 and ICAM4, the hypomethylated samples showed better prognosis compared with the hypermethylated samples $(\mathrm{P}<0.05)$. By contrast, DNASE1L1, ARF6, ITGB3 and C13orf16 had significantly longer OS time in the hypermethylated samples relative to the hypomethylated samples.

The association between the gene expression level of the nine predictive genes and prognosis was also underscored. Specifically, all samples in the training set were divided into a 
Table III. Methylation modules from weighted correlation network analysis.

\begin{tabular}{lccccc}
\hline $\begin{array}{l}\text { Module } \\
\text { color }\end{array}$ & $\begin{array}{c}\text { Count of } \\
\text { CpGs }\end{array}$ & $\begin{array}{c}\text { Correlation } \\
\text { efficient }\end{array}$ & $\mathrm{P}_{\text {corr }}$ & $\begin{array}{c}\text { Count of } \\
\text { DM CpGs }\end{array}$ & $\begin{array}{c}\text { Enrichment fold } \\
(95 \% \text { CI })\end{array}$ \\
\hline Black & 468 & 0.502 & 0.0024 & 68 & $2.818(2.106-3.728)$ \\
Blue & 368 & 0.609 & 0.0033 & 22 & $1.159(0.709-1.811)$ \\
Brown & 410 & 0.488 & 0.0047 & 10 & $0.473(0.223-0.889)$ \\
Green & 302 & 0.495 & 0.0027 & 18 & $1.156(0.668-1.886)$ \\
Yellow & 228 & 0.486 & 0.0023 & 8 & $0.681(0.288-1.379)$ \\
Grey & 3363 & 0.407 & 0.6550 & 117 & $0.674(0.541-0.837)$ \\
Magenta & 341 & 0.532 & 0.0007 & 17 & $0.967(0.550-1.594)$ \\
Pink & 299 & 0.469 & 0.0005 & 27 & $1.752(1.119-2.644)$ \\
Purple & 194 & 0.517 & 0.0068 & 10 & $0.999(0.468-1.901)$ \\
Red & 127 & 0.539 & 0.0005 & 1 & $0.153(0.038-0.872)$ \\
Salmon & 138 & 0.428 & 0.0039 & 26 & $3.653(2.275-5.673)$ \\
Tan & 127 & 0.471 & 0.0045 & 8 & 0.000176 \\
Turquoise & 343 & 0.811 & 0.0029 & 15 & 0.9680 \\
Yellow & 256 & 0.518 & 0.0007 & 12 & $0.848(0.465-1.439)$ \\
\end{tabular}

Count of CpGs, the number of CpGs included in one module; DM, differentially methylated; count of DM CpGs, the number of DM CpGs mapped to one module; $\mathrm{P}_{\text {corr }}$, P-value for correlation coefficient; $\mathrm{P}_{\text {hyper }}$ P-value for enrichment analysis; $\mathrm{CI}$, confidential interval.

Table IV. Significant GO terms for the three LUAD-associated modules.

\begin{tabular}{lrr}
\hline GO term & Count of genes & Fold enrichment \\
\hline Cytoskeleton organization & 8 & 2.821 \\
Response to DNA damage stimulus & 7 & 2.885 \\
Spindle organization & 3 & 10.248 \\
Positive regulation of cytoskeleton organization & 3 & 10.248 \\
Regulation of transcription from RNA polymerase II promoter & 10 & 2.115 \\
Cell junction organization & 3 & 8.091 \\
Anterior/posterior pattern formation & 4.392 \\
Regulation of cellular component biogenesis & 4 & 0.0169 \\
Microtubule cytoskeleton organization & 4 & 0.0169 \\
Positive regulation of transcription, DNA-dependent & 4 & 0.0218 \\
Positive regulation of RNA metabolic process & 7 & 0.0260 \\
Cell death & 7 & 0.0307 \\
Apoptosis & 9 & 0.0318 \\
Death & 8.256 \\
Programmed cell death & 2.237 \\
Positive regulation of organelle organization & 9 & 1.924 \\
\hline
\end{tabular}

GO, Gene Ontology; LUAD: lung adenocarcinoma; Count of genes, the number of genes significantly enriched in one GO term.

high- and low-expression group, according to the median expression level of each gene, separately. High expression of $C 20$ orf 56 , BTG2, ZDHHC3, FHDC1 and ICAM4 showed significantly improved prognosis in comparison with low expression samples (Fig. 5). Concerning DNASE1L1, ARF6, ITGB3 and C13orf16, improved survival was reported in the low-expression samples relative to high-expression samples (Fig. 5). For prognosis stratification, a prognostic scoring system was developed as follows:
Risk score $=(0.8529) \times$ methylation ${ }_{\mathrm{cg} 00933153}+(0.9931) \mathrm{x}$ methylation $_{\mathrm{cg} 01798157}+(-0.5670) \times$ methylation $_{\mathrm{cg} 01963754}+(-0.1602) \mathrm{x}$ methylation $_{\mathrm{cg} 04324559}+(0.6870) \mathrm{x}$ methylation ${ }_{\mathrm{cg} 04432377}+(1.0356) \mathrm{x}$ methylation $_{\mathrm{cg} 07097184}+(-0.9612) \times$ Methylation $_{\mathrm{cg} 10156217}+(-0.4113)$ $\mathrm{x}_{\text {methylation }} \mathrm{cg}_{\mathrm{cg} 10753610}+(0.4918) \mathrm{x}$ methylation cg21494776 $_{\text {. }}$

The risk score was calculated for each individual patient in the TCGA set accordingly. All patients in the TCGA set were classified into a high- and low-risk group by risk score using the 
Table V. Genes associated with survival according to Cox regression analysis.

\begin{tabular}{|c|c|c|}
\hline ID & Gene & P-value \\
\hline $\operatorname{cg} 03395898$ & TGFB3 & $6.0 \times 10^{-5}$ \\
\hline cg01798157 & BTG2 & $4.2 \times 10^{-4}$ \\
\hline $\operatorname{cg} 07097184$ & FHDC1 & $5.1 \times 10^{-4}$ \\
\hline $\operatorname{cg} 02334643$ & DHX40 & $8.4 \times 10^{-4}$ \\
\hline $\operatorname{cg} 05003322$ & COL16A1 & $8.6 \times 10^{-4}$ \\
\hline $\operatorname{cg} 27500918$ & FLYWCH2 & $9.1 \times 10^{-4}$ \\
\hline $\operatorname{cg} 00933153$ & C20orf56 & $1.0 \times 10^{-3}$ \\
\hline $\operatorname{cg} 20541456$ & CYFIP2 & $1.1 \times 10^{-4}$ \\
\hline $\operatorname{cg} 05898928$ & YPEL1 & $1.2 \times 10^{-4}$ \\
\hline $\operatorname{cg} 16194253$ & C14orf21 & $1.5 \times 10^{-4}$ \\
\hline $\operatorname{cg} 21494776$ & ICAM4 & $1.6 \times 10^{-4}$ \\
\hline cg11013977 & QRICH1 & $1.7 \times 10^{-4}$ \\
\hline $\operatorname{cg} 06620210$ & AP1M1 & $2.1 \times 10^{-4}$ \\
\hline $\operatorname{cg} 02016545$ & MICA & $2.3 \times 10^{-4}$ \\
\hline $\operatorname{cg} 03595580$ & TECPR1 & $2.5 \times 10^{-4}$ \\
\hline cg18050194 & SEC 22C & $4.0 \times 10^{-3}$ \\
\hline $\operatorname{cg} 08517562$ & PTPN1 & $5.6 \times 10^{-4}$ \\
\hline $\operatorname{cg} 02156071$ & C10orf84 & $5.7 \times 10^{-4}$ \\
\hline $\operatorname{cg} 06877599$ & SUPT5H & $5.7 \times 10^{-4}$ \\
\hline $\operatorname{cg} 04902921$ & EDEM3 & $6.3 \times 10^{-4}$ \\
\hline $\operatorname{cg} 16740905$ & SEC1 & $6.9 \times 10^{-4}$ \\
\hline $\operatorname{cg} 04432377$ & ZDHHC3 & $7.1 \times 10^{-4}$ \\
\hline $\operatorname{cg} 10753610$ & ITGB3 & $7.9 \times 10^{-4}$ \\
\hline $\operatorname{cg} 05714082$ & POLR1A & $8.5 \times 10^{-4}$ \\
\hline $\operatorname{cg} 04446303$ & TFAP4 & $9.4 \times 10^{-4}$ \\
\hline $\operatorname{cg} 02282317$ & AATF & 0.011 \\
\hline $\operatorname{cg} 08100565$ & SLC25A36 & 0.011 \\
\hline $\operatorname{cg} 01889020$ & MEGF11 & 0.014 \\
\hline cg02181309 & MRPL52 & 0.014 \\
\hline $\operatorname{cg} 01498883$ & SNRPB & 0.014 \\
\hline $\operatorname{cg} 06677352$ & STAG3L3 & 0.014 \\
\hline $\operatorname{cg} 24122247$ & CIDECP & 0.015 \\
\hline $\operatorname{cg} 24135606$ & PFN1 & 0.016 \\
\hline $\operatorname{cg} 24612420$ & ACLY & 0.017 \\
\hline cg19759282 & GPR155 & 0.017 \\
\hline cg13939431 & MEAF6 & 0.017 \\
\hline $\operatorname{cg} 01963754$ & C13orf16 & 0.018 \\
\hline $\operatorname{cg} 04324559$ & DNASE1L1 & 0.019 \\
\hline $\operatorname{cg} 24951800$ & MEF2A & 0.019 \\
\hline $\operatorname{cg} 00567190$ & C1orf97 & 0.020 \\
\hline $\operatorname{cg} 12811419$ & TMEM214 & 0.024 \\
\hline cg00399374 & CHMP4C & 0.025 \\
\hline cg01779512 & IFT88 & 0.027 \\
\hline $\operatorname{cg} 16931807$ & KIAA0195 & 0.032 \\
\hline $\operatorname{cg} 15599146$ & ZDHHC14 & 0.033 \\
\hline $\operatorname{cg} 07459266$ & RNF213 & 0.034 \\
\hline $\operatorname{cg} 08125503$ & PIGL & 0.035 \\
\hline $\operatorname{cg} 07057042$ & RAB5A & 0.035 \\
\hline cg21994174 & ETFB & 0.036 \\
\hline $\operatorname{cg} 06421633$ & LUC7L3 & 0.036 \\
\hline $\operatorname{cg} 03270167$ & RAMP1 & 0.037 \\
\hline
\end{tabular}

Table V. Continued.

\begin{tabular}{lll}
\hline ID & Gene & P-value \\
\hline $\operatorname{cg} 05006947$ & SLC38A7 & 0.040 \\
$\operatorname{cg} 10156217$ & ARF6 & 0.042 \\
$\operatorname{cg} 07097417$ & LPGAT1 & 0.042 \\
$\operatorname{cg} 02146453$ & PROSC & 0.043 \\
$\operatorname{cg} 17286258$ & SF3B1 & 0.043 \\
$\operatorname{cg} 18085070$ & PSRC1 & 0.044 \\
$\operatorname{cg} 27229100$ & C20orf199 & 0.046 \\
\hline
\end{tabular}

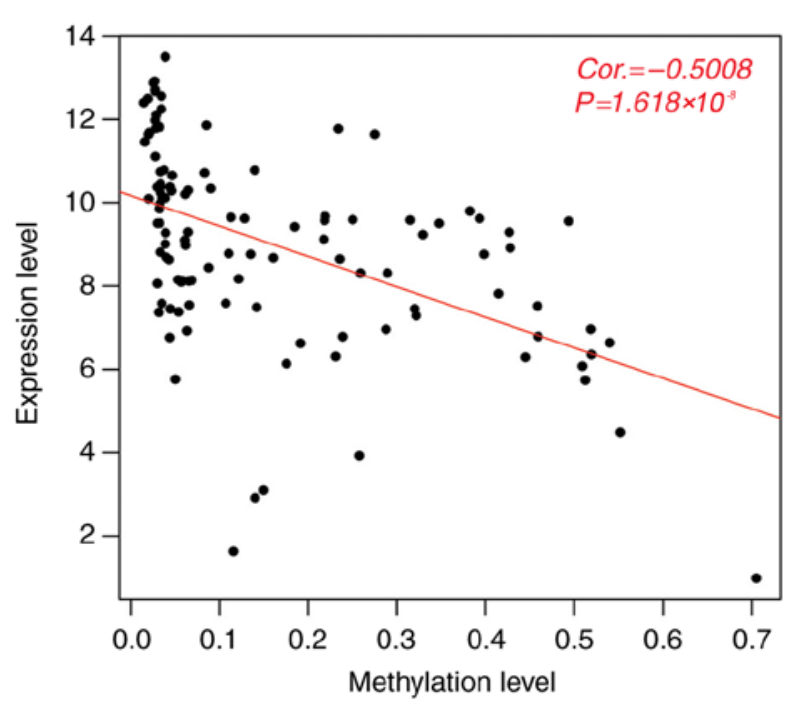

Figure 3. A scatter diagram for correlation analysis between the gene methylation and expression levels in the three lung adenocarcinoma-associated modules. Black dots represent the genes. The red line represents the trend line of the data points. Cor, Pearson's correlation coefficient.

median risk score of all patients as the cut-off value. As shown in Fig. 6A, low-risk patients had significantly longer OS time compared with the high-risk patients $\left(\mathrm{P}=8.992 \times 10^{-7}\right)$, with an AUC value of 0.966 . The nine-gene methylation signature was also applied to predict the patients' probability of recurrence in the training set. The low-risk patients had visibly longer RFS time in comparison with the high-risk patients, with an AUC value of 0.928 (Fig. 6B). In order to test the capability of the nine-gene methylation signature, all samples in the validation set were classified by risk score into high- and low-risk groups. Similarly, low-risk patients had a dramatically longer RFS time relative to high-risk patients. The AUC value was 0.920 (Fig. 6C), confirming the prognostic power of the nine-gene methylation signature.

\section{Discussion}

LUAD is clinicopathologically and molecularly heterogeneous, making the prediction of patient outcome a necessity $(22,23)$. Gene silencing at the epigenetic level by DNA methylation was acknowledged as an important mechanism underlying tumorigenesis (24). The present study placed 
Table VI. A panel of nine differentially methylated genes predictive of prognosis.

\begin{tabular}{llcclccr}
\hline ID & Gene & Chromosome & Position & Location & Coefficient & Hazard ratio & P-value \\
\hline cg00933153 & C20orf56 & 20 & 22498129 & Body & 0.8529 & 3.177 & 0.0010 \\
cg01798157 & BTG2 & 1 & 201543218 & Promoter & 0.9931 & 2.606 & 0.0004 \\
cg01963754 & C13orf16 & 13 & 110775249 & Body & -0.5670 & 0.499 & 0.0180 \\
$\operatorname{cg} 04324559$ & DNASE1L1 & X & 153290455 & 5'UTR & -0.1602 & 0.712 & 0.0190 \\
cg04432377 & ZDHHC3 & 3 & 44992301 & Promoter & 0.6870 & 2.894 & 0.0071 \\
cg07097184 & FHDC1 & 4 & 154119462 & 3'UTR & 1.0356 & 5.175 & 0.0005 \\
cg10156217 & ARF6 & 14 & 49432104 & Promoter & -0.9612 & 0.260 & 0.0420 \\
cg10753610 & ITGB3 & 17 & 42690201 & Body & -0.4113 & 0.489 & 0.0079 \\
$\operatorname{cg} 21494776$ & ICAM4 & 19 & 10258780 & Body & 0.4918 & 2.065 & 0.0016 \\
\hline
\end{tabular}

cg00933153/C20orf56

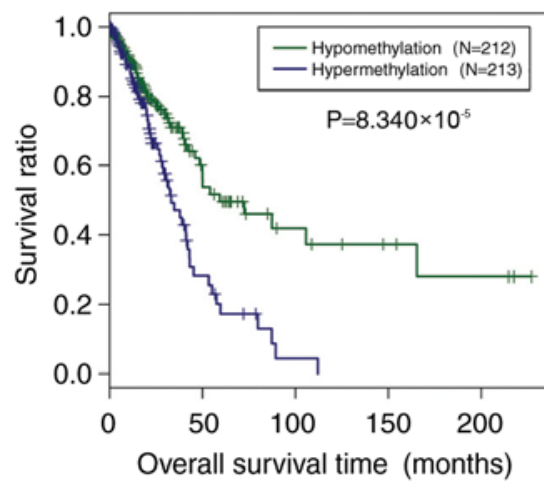

cg07097184/FHDC1

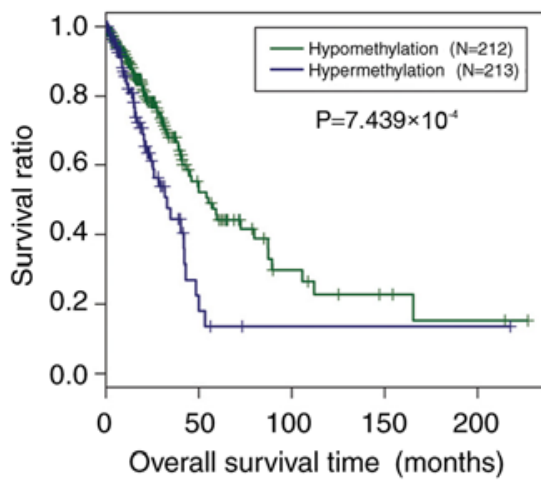

cg10156217/ARF6

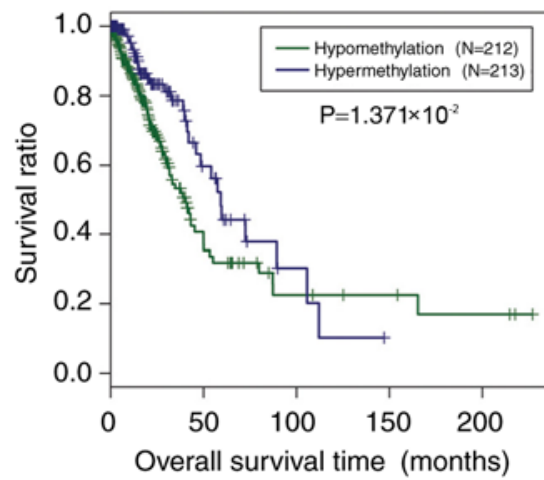

cg01798157/BTG2

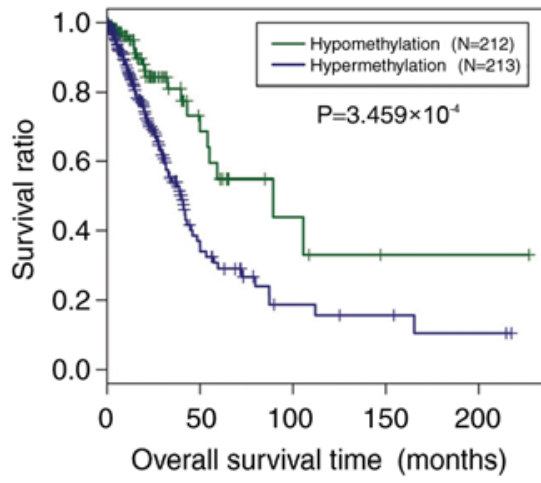

cg21494776/ICAM4

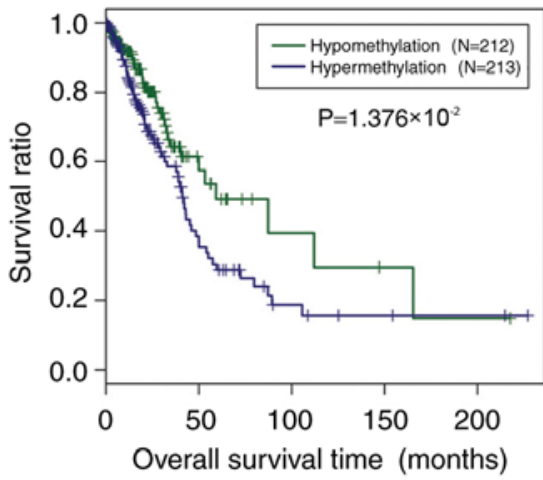

cg10753610/ITGB3

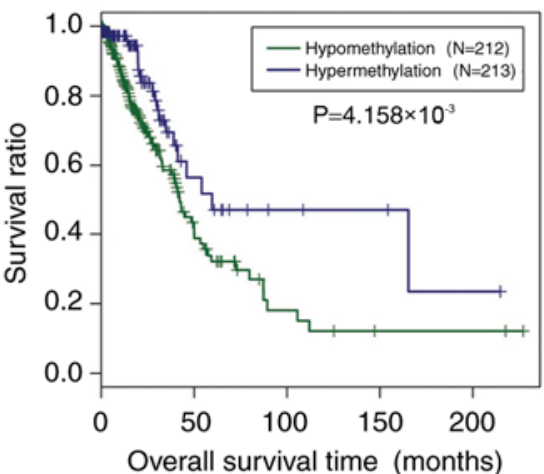

cg04432377/ZDHHC3

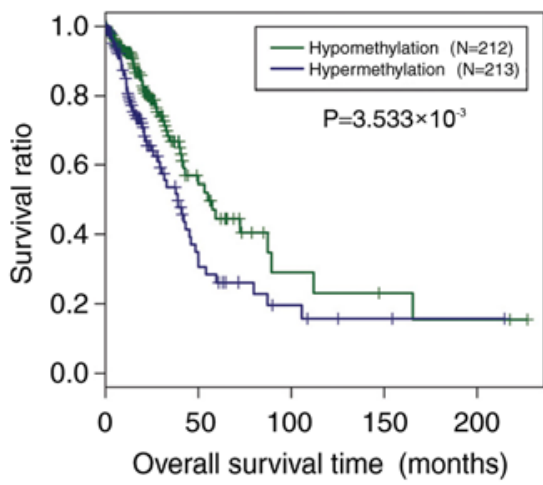

cg04324559/DNASE1L1

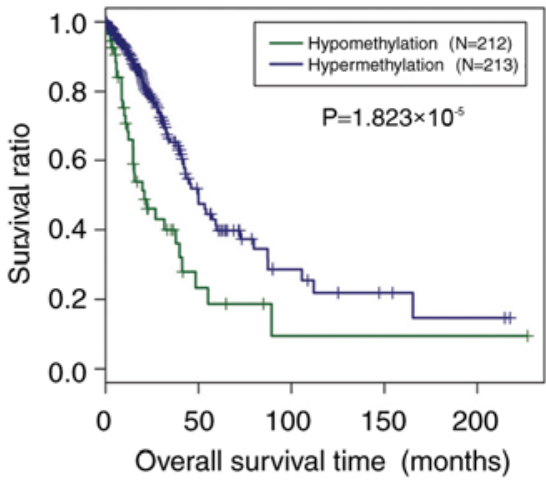

cg01963754/C13orf16

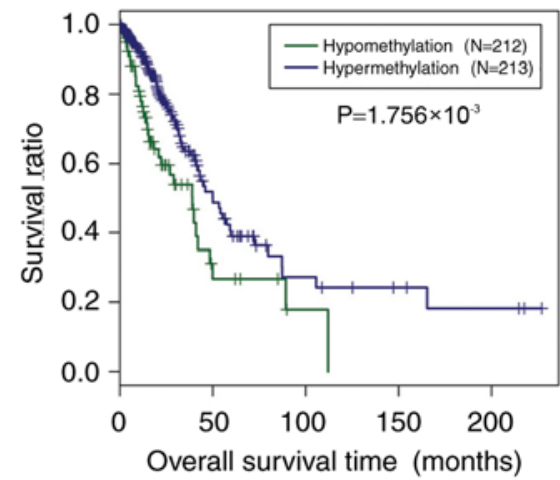

Figure 4. Kaplan-Meier curves of dichotomized patients, based on the methylation level of each gene in the training set. According to the methylation level of one gene, all patients in the training set were separated into a hypermethylation and a hypomethylation group. 
C20orf56

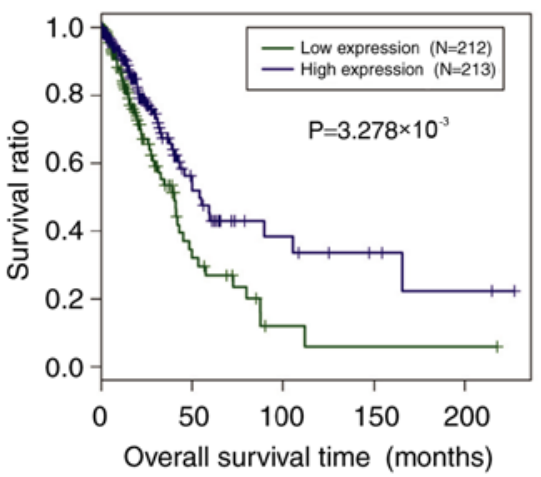

FHDC1

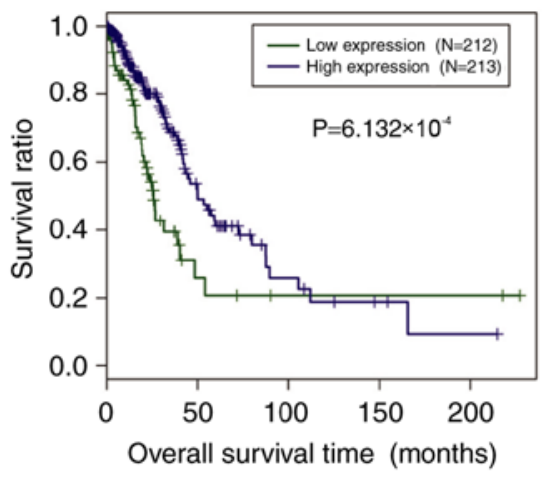

ARF6

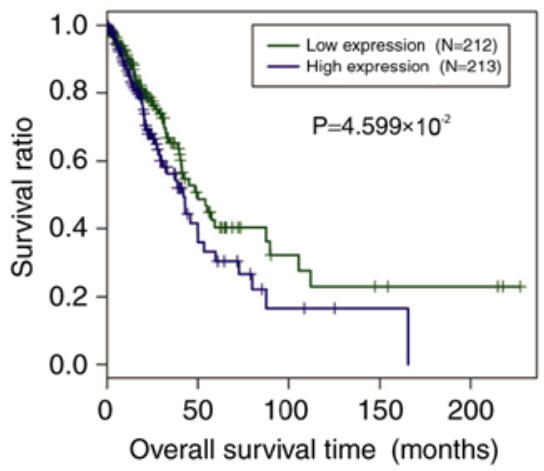

BTG2

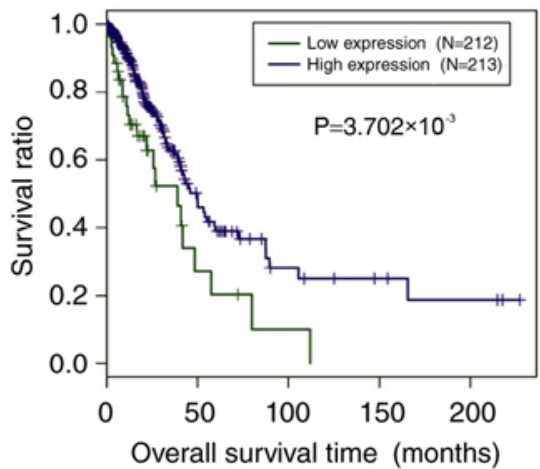

ICAM4

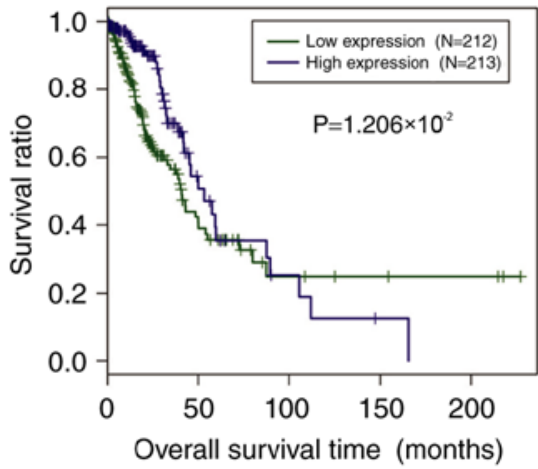

ITGB3

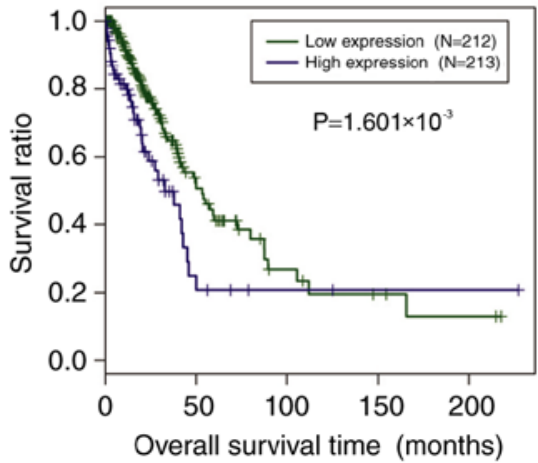

ZDHHC3

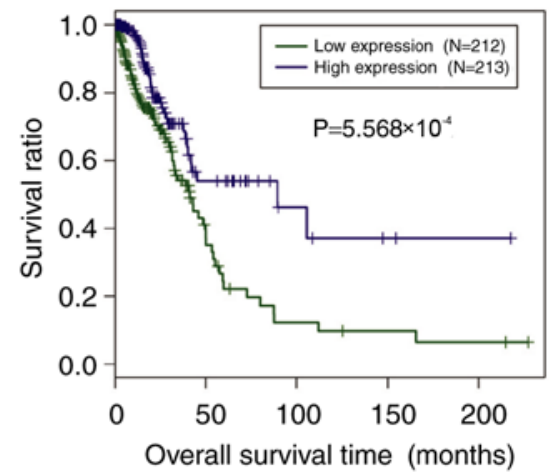

DNASE1L1

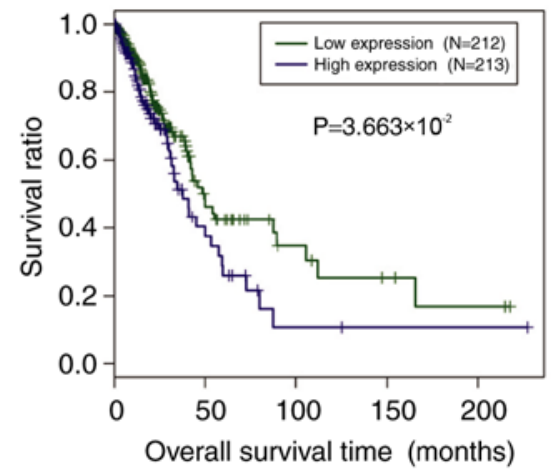

C13orf16

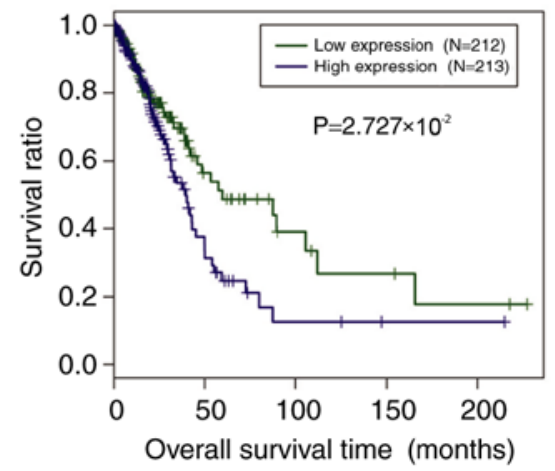

Figure 5. Kaplan-Meier curves showing patients, classified based on the expression level of each gene in the training set. Based on the gene expression level of one gene, all patients in the training set are classified into a high and low expression group.

a special focus on DNA methylation alterations in LUAD and their implications for prognosis. A total of 742 DMGs were identified, showing significantly different methylations in CpGs between the samples designated as good and bad prognosis. There were three LUAD-associated DNA co-methylation modules mined by WGCNA. The 121 DMGs in the 3 modules were significantly associated with $16 \mathrm{GO}$ terms. Notably, three GO terms were associated with cytoskeleton organization, two GO terms with transcription and four GO terms with cell apoptosis or death. These findings provided some insight into the underlying mechanisms of DNA methylation alterations in LUAD.

The present study identified a nine-gene methylation signature from the genes included in the three LUAD-associated DNA methylation modules. Moreover, risk score derived from the sum of each candidate methylation marker multiplied by the corresponding regression coefficient successfully classified patients into two risk groups, with significantly different OS or RFS time. The prognostic performance of this nine-gene signature was successfully verified for RFS time in the validation set. These findings indicate that the nine genes were valuable methylation markers for prognostic evaluation in LUAD patients.

The nine novel candidate methylation markers for prognosis prediction included C20orf56, BTG2, C13orf16, DNASE1L1, ZDHHC3, FHDC1, ARF6, ITGB3 and ICAM4. Moreover, the present study found that hypomethylation/high expression of ICAM4, ZDHHC3, C20orf56, BTG2 and FHDCl was associated with significantly improved survival outcome compared with hypermethylation/low expression. In contrast, hypermethylation/low expression of DNASE1L1, ARF6, ITGB3 and C13orf16 corresponded with significantly improved survival 
A

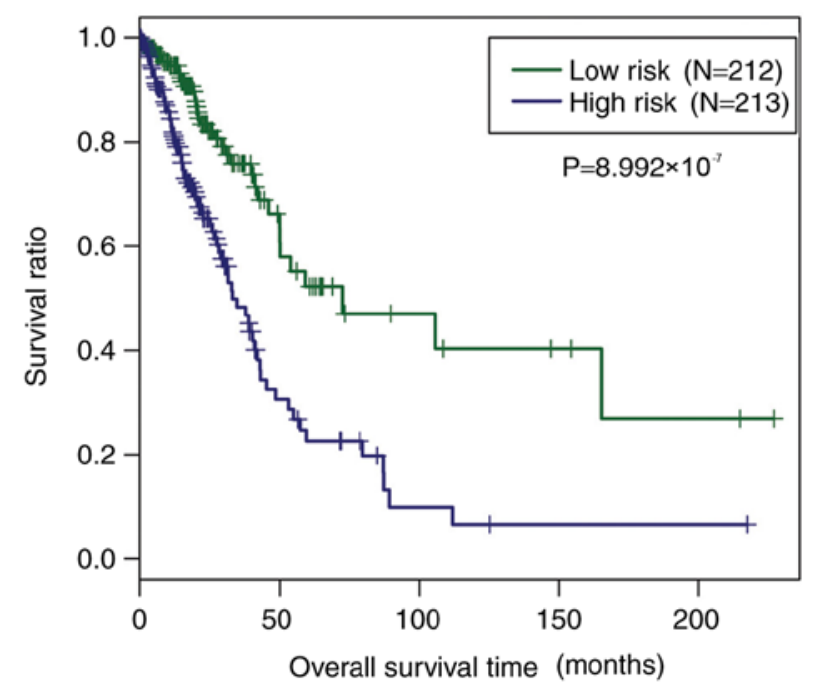

B

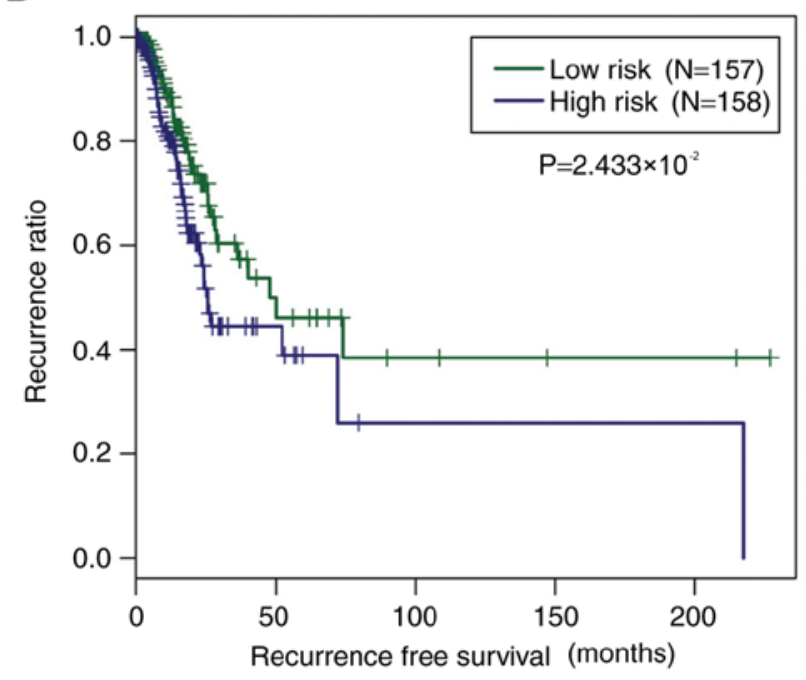

C

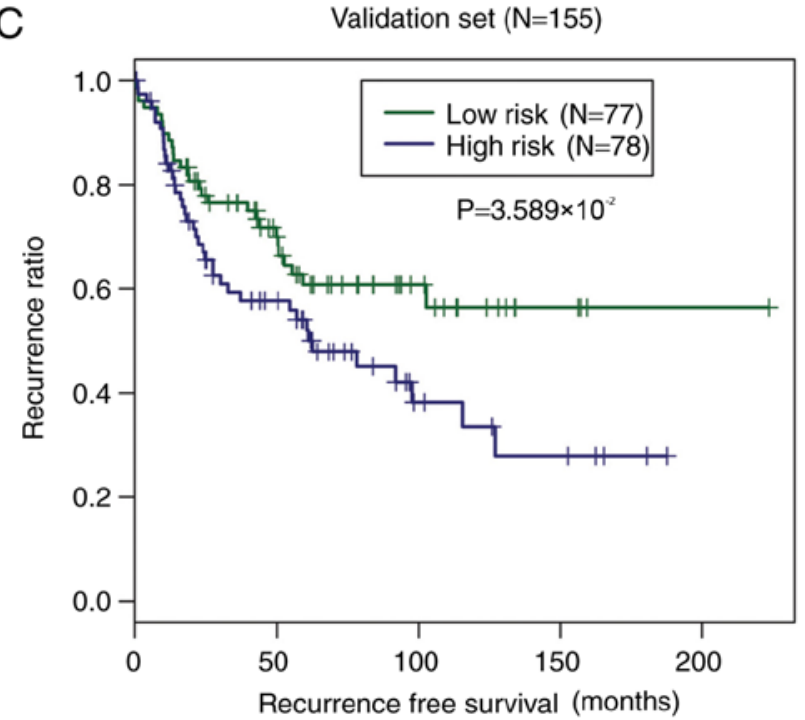

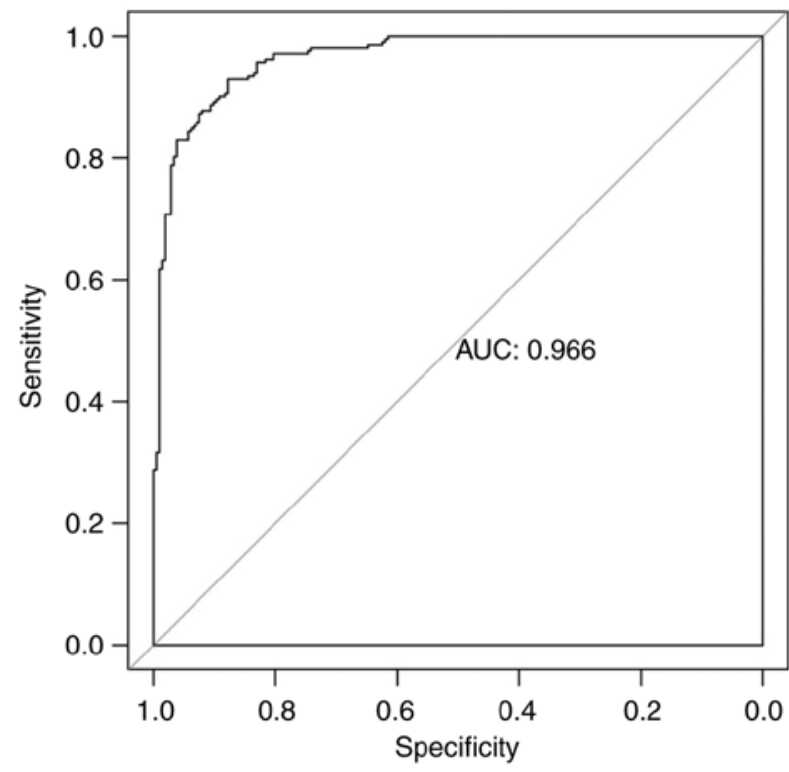
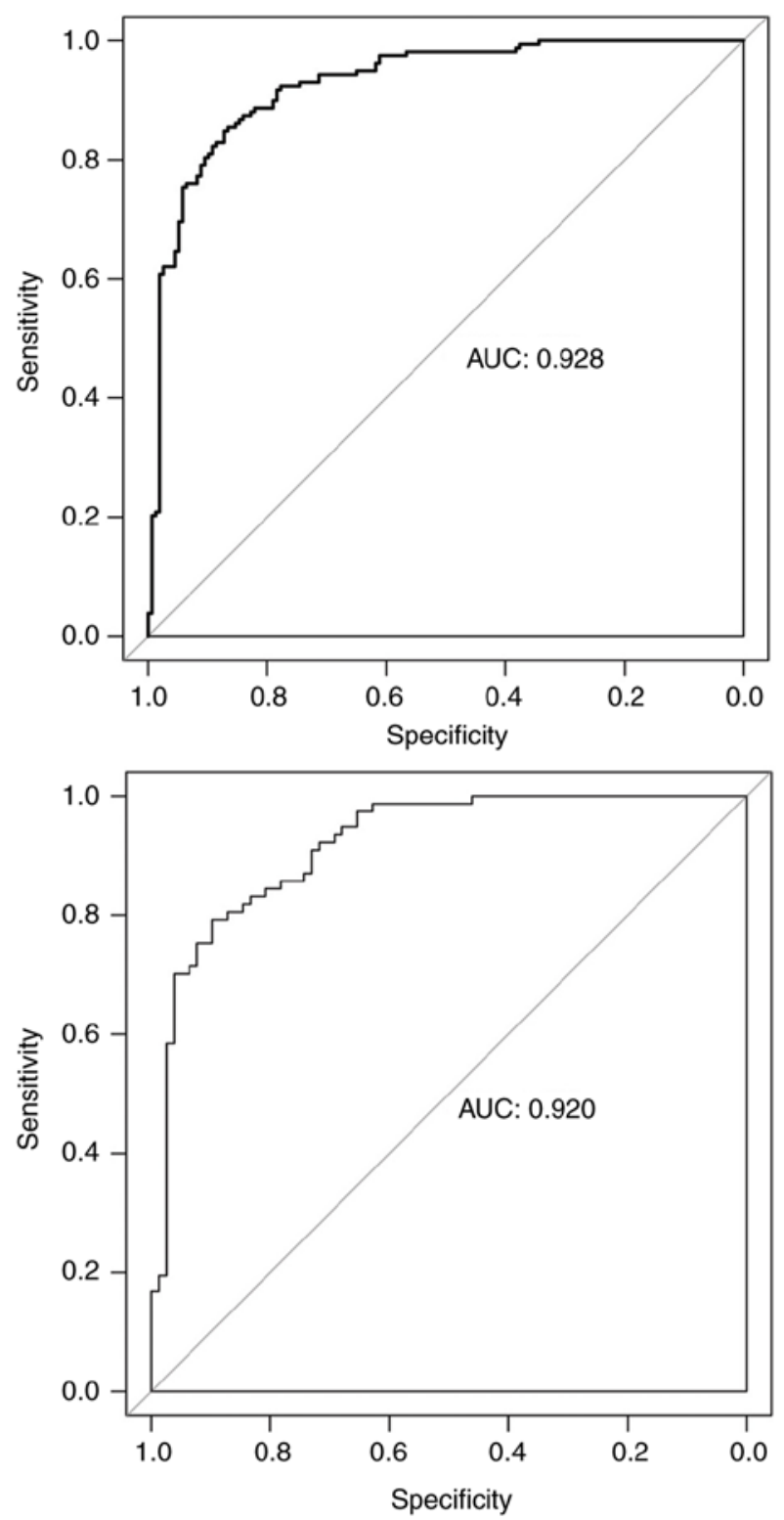

Figure 6. Kaplan-Meier curves (left panel) and ROC curves (right panel) demonstrating patients assigned to two risk groups, based on the nine-gene methylation signature in the (A and B) training set and the (C) validation set. (A) Overall survival time and (B) recurrence-free survival time of the high- and low-risk groups of the training set were estimated using Kaplan-Meier curves. The AUC of ROC reveals the capability of the nine-gene methylation signature to predict survival or recurrence. ROC, receiver operating characteristic; AUC, area under the curve. 
in comparison with when these genes were hypomethylated/highly expressed.

$B T G 2$ is involved in various biological activities, such as cell differentiation, proliferation and apoptosis, and has been acknowledged as a tumor suppressor in several types of cancer in humans, including laryngeal carcinoma, gastric cancer, clear cell renal cell carcinoma and breast carcinoma (25-29). More importantly, there is evidence that $B T G 2$ overexpression suppressed the growth and proliferation of lung cancer cells (30). It can be speculated that aberrant BTG2 methylation participated in the regulation of genes involved in LUAD tumor growth. In addition, the present study implicated two possible functions for BTG2: Response to DNA damage stimulus and anterior/posterior pattern formation in LUAD. These findings offer useful information concerning the role of BTG2 through the regulation of methylation in LUAD.

$A R F 6$ is a member of the human $A R F$ gene family and plays a role in vesicular trafficking. Increasing evidence demonstrated an association between ARF6 and tumor cell invasion (31,32). Moreover, in the present study, GO analysis showed that ARF6 was significantly implicated in the positive regulation of cytoskeleton organization, regulation of cellular component biogenesis, cell death, apoptosis and positive regulation of organelle organization. Thus, aberrant ARF6 methylation may play a role in the regulation expression of genes associated with these biological processes in LUAD.

ITGB3, also known as CD61, is a protein encoded by ITGB3 and participates in cell adhesion and signaling mediated by the cell surface $(33,34)$. The present study found that ITGB3 was significantly enriched in cell junction organization in LUAD. Based on these observations, it can be inferred that alteration of ITGB3 methylation exerted an effect on the expression of genes involved in cell junction organization, thus modulating cell adhesion and cell surface-mediated signaling. ICAM4 is a member of the ICAMs family and is critical for inflammation and immune responses (35). DNASE1L1 is an enzyme encoded by DNASE1L1, a member of the human DNase family. To the best of our knowledge, there are few reports regarding the function of ZDHHC3, C13orf16, C20orf56, FHDC1, ICAM4 and DNASE1L1 in LUAD.

The limitations of the present study include minimal information on the OS time in the validation set, as other DNA methylation dataset of LUAD with survival information could not be located in NCBI GEO. Furthermore, only correlations between DNA methylation and the gene expression levels were investigated, based on data of LUAD downloaded from TCGA. Thus, protein expression of the nine novel candidate methylation markers for prognostic prediction should be also studied in the future. The aim of the present study was to provide novel prognostic DNA methylation biomarkers of LUAD, since the modern precise medicine for the treatment of LUAD required additional biomarkers and novel therapeutic targets of interest. However, the findings of the present study require validation in prospective clinical trials before the prognostic multigene methylation signature can be applied. Therefore, Chinese-population-based validation could be considered in the future.

In conclusion, the present study focused on the DNA methylation changes associated with LUAD and identified a prognostic nine-gene methylation signature for LUAD. The findings shed light on the DNA methylation landscape in
LUAD and its implications on the development of optimized and individualized management of this condition.

\section{Acknowledgements}

Not applicable.

\section{Funding}

No funding was received.

\section{Availability of data and materials}

The datasets used and/or analyzed during the present study are available from the corresponding author on reasonable request.

\section{Authors' contributions}

RW and HZ analyzed and interpreted the sequencing data. MXY conceived and designed the study. CRZ checked the data analysis results and was a major contributor in writing the manuscript. All authors read and approved the final manuscript.

\section{Ethics approval and consent to participate}

Not applicable.

\section{Patient consent for publication}

Not applicable.

\section{Competing interests}

The authors declare that they have no competing interests.

\section{References}

1. Zhou C: Lung cancer molecular epidemiology in China: Recent trends. Transl Lung Cancer Res 3: 270-279, 2014.

2. Stewart BW and Wild CP (eds): World Cancer Report 2014. IARC, Lyon, 2014.

3. Imielinski M, Berger AH, Hammerman PS, Hernandez B, Pugh TJ, Hodis E, Cho J, Suh J, Capelletti M, Sivachenko A, et al: Mapping the hallmarks of lung adenocarcinoma with massively parallel sequencing. Cell 150: 1107-1120, 2012

4. Reck M,Heigener DF,Mok T, Soria JC and Rabe KF: Management of non-small-cell lung cancer: Recent developments. Lancet 382: 709-719, 2013.

5. Goodgame B, Viswanathan A, Miller CR, Gao F, Meyers B, Battafarano RJ, Patterson A, Cooper J, Guthrie TJ, Bradley J, et al: A clinical model to estimate recurrence risk in resected stage I non-small cell lung cancer. Am J Clin Oncol 31: 22-28, 2008.

6. Robertson KD: DNA methylation and human disease. Nat Rev Genet 6: 597-610, 2005.

7. Kerr KM, Galler JS, Hagen JA, Laird PW and Laird-Offringa IA: The role of DNA methylation in the development and progression of lung adenocarcinoma. Dis Markers 23: 5-30, 2007.

8. Zhu XF, Zhu BS, Wu FM and Hu HB: DNA methylation biomarkers for the occurrence of lung adenocarcinoma from TCGA data mining. J Cell Physiol 233: 6777-6784, 2018.

9. Han L, Xu G, Xu C, Liu B and Liu D: Potential prognostic biomarkers identified by DNA methylation profiling analysis for patients with lung adenocarcinoma. Oncol Lett 15: 3552-3557, 2018.

10. Sandoval J, Mendez-Gonzalez J, Nadal E, Chen G, Carmona FJ, Sayols S, Moran S, Heyn H, Vizoso M, Gomez A, et al: A prognostic DNA methylation signature for stage I non-small-cell lung cancer. J Clin Oncol 31: 4140-4147, 2013. 
11. Kuo IY, Jen J, Hsu LH, Hsu HS, Lai WW and Wang YC: A prognostic predictor panel with DNA methylation biomarkers for early-stage lung adenocarcinoma in Asian and Caucasian populations. J Biomed Sci 23: 58, 2016.

12. Ritchie ME, Phipson B, Wu D, Hu Y,Law CW, Shi W and Smyth GK: Limma powers differential expression analyses for RNA-sequencing and microarray studies. Nucleic Acids Res 43: e47, 2015.

13. Langfelder $P$ and Horvath S: WGCNA: An R package for weighted correlation network analysis. BMC Bioinformatics 9: 559, 2008.

14. Cao J and Zhang S: A bayesian extension of the hypergeometric test for functional enrichment analysis. Biometrics 70: 84-94, 2014.

15. Huang da W, Sherman BT and Lempicki RA: Bioinformatics enrichment tools: Paths toward the comprehensive functional analysis of large gene lists. Nucleic Acids Res 37: 1-13, 2009.

16. Huang da W, Sherman BT and Lempicki RA: Systematic and integrative analysis of large gene lists using DAVID bioinformatics resources. Nat Protoc 4: 44-57, 2009.

17. Eberly LE: Correlation and simple linear regression. Methods Mol Biol 404: 143-164, 2007.

18. Wang P, Wang Y, Hang B, Zou X and Mao JH: A novel gene expression-based prognostic scoring system to predict survival in gastric cancer. Oncotarget 7: 55343-55351, 2016.

19. Tibshirani R: The lasso method for variable selection in the Cox model. Stat Med 16: 385-395, 1997.

20. Goeman JJ: L1 penalized estimation in the Cox proportional hazards model. Biom J 52: 70-84, 2010.

21. Goel MK, Khanna P and Kishore J: Understanding survival analysis: Kaplan-Meier estimate. Int J Ayurveda Res 1: 274-278, 2010.

22. Chen Z, Fillmore CM, Hammerman PS, Kim CF and Wong KK Non-small-cell lung cancers: A heterogeneous set of diseases. Nat Rev Cancer 14: 535-546, 2014.

23. Greulich $\mathrm{H}$ : The genomics of lung adenocarcinoma: Opportunities for targeted therapies. Genes Cancer 1: 1200-1210, 2010.

24. Momparler RL and Bovenzi V: DNA methylation and cancer. J Cell Physiol 183: 145-154, 2015.

25. Mao B, Zhang Z and Wang G: BTG2: A rising star of tumor suppressors (review). Int J Oncol 46: 459-464, 2015.

26. Liu M, Wu H, Liu T, Li Y, Wang F, Wan H, Li X and Tang $\mathrm{H}$ : Regulation of the cell cycle gene, BTG2, by miR-21 in human laryngeal carcinoma. Cell Res 19: 828-837, 2009.
27. Struckmann K, Schraml P, Simon R,Elmenhorst K, Mirlacher M, Kononen $\mathrm{J}$ and Moch $\mathrm{H}$ : Impaired expression of the cell cycle regulator BTG2 is common in clear cell renal cell carcinoma. Cancer Res 64: 1632-1638, 2004

28. Takahashi F, Chiba N, Tajima K, Hayashida T, Shimada T, Takahashi M, Moriyama H, Brachtel E, Edelman EJ, Ramaswamy S and Maheswaran S: Breast tumor progression induced by loss of BTG2 expression is inhibited by targeted therapy with the ErbB/HER inhibitor lapatinib. Oncogene 30: 3084-3095, 2011.

29. Zhang L, Huang H, Wu K, Wang M and Wu B: Impact of BTG2 expression on proliferation and invasion of gastric cancer cells in vitro. Mol Biol Rep 37: 2579-2586, 2010.

30. Wei S, Hao C, Xin L, Zhao H, Chen J and Zhou Q: Effects of BTG2 on proliferation inhibition and anti-invasion in human lung cancer cells. Tumour Biol 33: 1223-1230, 2012.

31. Tague SE, Muralidharan V and D'Souza-Schorey C: ADP-ribosylation factor 6 regulates tumor cell invasion through the activation of the MEK/ERK signaling pathway. Proc Natl Acad Sci USA 101: 9671-9676, 2004.

32. Muralidharan-Chari V, Hoover H, Clancy J, Schweitzer J, Suckow MA, Schroeder V, Castellino FJ, Schorey JS and D'Souza-Schorey C: ADP-ribosylation factor 6 regulates tumorigenic and invasive properties in vivo. Cancer Res 69: 2201-2209, 2009.

33. Seguin L, Desgrosellier JS, Weis SM and Cheresh DA: Integrins and cancer: Regulators of cancer stemness, metastasis, and drug resistance. Trends Cell Biol 25: 234-240, 2015.

34. Desgrosellier JS and Cheresh DA: Integrins in cancer: Biological implications and therapeutic opportunities. Nat Rev Cancer 10: 9-22, 2010.

35. Gahmberg CG, Tolvanen M and Kotovuori P: Leukocyte adhesion-structure and function of human leukocyte beta2-integrins and their cellular ligands. Eur J Biochem 245: 215-232, 1997.

This work is licensed under a Creative Commons Attribution-NonCommercial-NoDerivatives 4.0 International (CC BY-NC-ND 4.0) License. 